\title{
Minimal Waiting Times in Static Traffic Control
}

\author{
O. MOESCHLIN $\dagger$ \\ otto.moeschlin@fernuni-hagen.de \\ Department of Mathematics, University of Hagen, D-58084 Hagen \\ C. POPPINGA \\ carsten.poppinga@fernuni-hagen.de \\ Department of Mathematics, University of Hagen, D-58084 Hagen
}

\begin{abstract}
The paper discusses the question of the optimal control of an unsymmetric bottleneck system with Poisson arrival processes having the minimization of the mean individual waiting time as objective. The setup allows the straightforward generalization to more complicated forms of traffic organization. The notion of the mean individual waiting time is based on a theorem of the Little type, which is derived by a strong law of large numbers. The proof makes use of McNeil's formula, which connects the expected total waiting time with the expected queue length.
\end{abstract}

Keywords: Bottleneck system, Poisson arrival process, Markov chain, a strong law of large numbers, a theorem of the Little type, minimal individual waiting time, maximal throughput per time unit.

\section{Introduction}

In [2] and [3] the control of traffic lights at a bottleneck was - to our knowledge - treated for the first time. The solutions of such problems are of special practical importance. As in [2] and [3] the symmetric case is studied, the asymptotic expected queue length could be taken there as objective function.

With regard to more complicated forms of traffic organization, i.e. junctions or roundabouts, the solution of the unsymmetric bottleneck problem is required, where the optimality definition from [3] fails.

Decisive for the further development to the theory of the control of traffic lights is an adequate optimality principle, as which we take now the minimization of the mean individual waiting time.

This concept seems to be even meaningful for a generalization to a dynamic control of traffic lights, i.e. to a control of traffic on demand.

$\dagger$ Requests for reprints should be sent to O. Moeschlin,Department of Mathematics, University of Hagen, D-58084 Hagen, Germany. 
The set-up in [2] and [3] is based on the Lindley-recursion for the discrete time case. Rather than to appeal to standard queueing methods and results, the proof for ergodicity in [3] is given by a fixed-point theorem applied to a set of probability measures. One reason for the chosen approach was the fact, that not only the weak convergence but also the finiteness of the asymptotic expectation of the queue length had to be established.

The present approach is - by contrast - based on a strong law of large numbers as well as a theorem of the Little type adapted to the present case. The approach presented here is - of course - related to queueing and Markov theory, but is not covered by it.

The proving approach is insofar meaningful as all our computer experiments are justified by this law of large numbers.

\section{Model Description and Basic Definitions}

Traffic lights at a bottleneck give mutually free course to at most one of the two traffic streams from the both sides of the bottleneck. A typical example for such a bottleneck situation is a two lane road (one lane for each direction) with one lane being under construction, so that the vehicles from the both sides have to share the one remaining lane, cp. Figure 1.

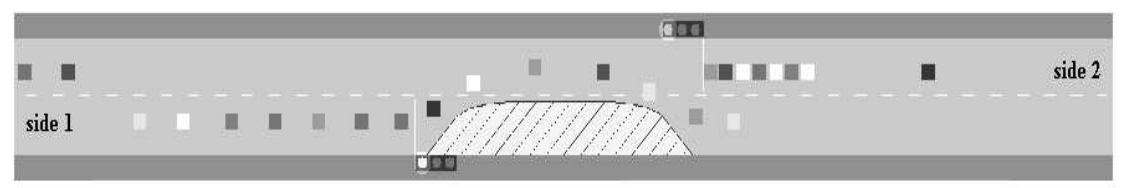

Figure 1. The vehicles from the left hand side, say side 1, have free passage, while the vehicles from side 2 (right hand side) have to wait.

Notice, the bottleneck situation is prototypical for more complicated forms of traffic organization. To describe the technical part of the bottleneck controlled by traffic lights for the unsymmetric case, the following parameters are used:

$$
\Delta_{i}, t_{R i}, \quad i=1,2 .
$$

$\Delta_{i}$ in $[\mathrm{veh} / \mathrm{s}]$ is the passage capacity (the maximal possible flow) for side $i, i=1,2$. $t_{R i}$ in $[\mathrm{s}]$ denotes the clearance time for side $i, i=1,2$, this is the time a vehicle needs to pass the bottleneck. The arrival processes 
$A^{(i)}=\left(A_{t}^{(i)}\right)_{t \in \mathbb{R}}$ for the both sides are assumed as independent Poisson processes on the probability space $(\Omega, \mathcal{A}, P)$ with parameter $I_{i}$ being the traffic intensity in $[\mathrm{veh} / \mathrm{s}]$ in a traffic-theoretic interpretation.

For $0 \leq s \leq t$ let

$$
N^{(i)}((s, t]):=A_{t}^{(i)}-A_{s}^{(i)}
$$

be the increment of the arrival process $A^{(i)}$ during the time interval $(s, t]$ for $i=1,2$. Moreover, let $q_{0}^{(i)}$ be the initial distribution of the number of vehicles waiting on side $i=1,2$ has to be introduced. The system of the bottleneck controlled by traffic lights may be comprised in an 8-tuple

$$
B:=\left(\Delta_{1}, \Delta_{2}, t_{R 1}, t_{R 2}, I_{1}, I_{2}, q_{0}^{(1)}, q_{0}^{(2)}\right),
$$

which is called a bottleneck experiment. To a given bottleneck experiment the times $t_{F i}>0$ of open passage (signalized by GREEN and afterwards by YELLOW) are the control variables (in the hand of the installation administrator). The duration of the phase of closed passage for side $i$ is given by

$$
t_{C i}:=t_{R 1}+t_{R 2}+t_{F(3-i)}, \quad i=1,2,
$$

while

$$
t_{U}:=t_{R 1}+t_{F 1}+t_{R 2}+t_{F 2}
$$

represents the length of a full control period. The function $\bar{\alpha}_{i}: \mathbb{R}_{+} \rightarrow \mathbb{Z}_{+}$ is defined by

$$
\bar{\alpha}_{i}(t):=\left\{\begin{array}{cc}
0 & , \quad 0<t<t_{C i} \\
{\left[\left(t-t_{C i}\right) \cdot \Delta_{i}\right]} & , t_{C i} \leq t \leq t_{U}
\end{array}\right.
$$

and the condition that $\bar{\alpha}_{i}$ is periodic with period $t_{U}$ on $\mathbb{R}_{+}, i=1,2 . \bar{\alpha}_{i}(t)$ represents the maximal number of vehicles which can pass the bottleneck from the beginning of a control period until the time $t$ of the control period.

(Notice that $[a]$ means the greatest integer number less than or equal to a.)

The number

$$
\alpha_{i}\left(t_{F i}\right):=\left[t_{F i} \cdot \Delta_{i}\right]=\bar{\alpha}_{i}\left(t_{U}\right)
$$

denotes the maximal number of vehicles that may pass the bottleneck from side $i$ during one control period, $i=1,2$.

Let $L_{0}^{(i)}:(\Omega, \mathcal{A}, P) \rightarrow \mathbb{Z}_{+}$be a random variable having the distribution $q_{0}^{(i)}$, independent of the arrival process $A^{(i)}, i=1,2$. 
The process $\left(L^{(i)}(t)\right)_{t \in \mathbb{R}}$ of queue lengths (of vehicles) on side $i$ is recursively defined by the Lindley equation

$$
\begin{gathered}
L^{(i)}(0):=L_{0}^{(i)} \\
L^{(i)}\left((j+1) t_{U}\right)=\begin{array}{l}
\left(L^{(i)}\left(j t_{U}\right)+N^{(i)}\left(\left(j t_{U},(j+1) t_{U}\right]\right)\right. \\
\left.-\alpha_{i}\left(t_{F i}\right)\right)_{+}
\end{array}
\end{gathered}
$$

and by

$$
L^{(i)}(t)=\left(L^{(i)}\left(j t_{U}\right)+N^{(i)}\left(\left(j t_{U}, t\right]\right)-\bar{\alpha}_{i}(t)\right)_{+}
$$

for $j t_{U}<t<(j+1) t_{U}(j=0,1, \ldots)$ and for $i=1,2$, where $(z)_{+}$stands for the positive part $\max \{z, 0\}$ of the number $z$. Define

$$
\lambda_{i}\left(t_{F 1}, t_{F 2}\right):=I_{i} \cdot\left(t_{F 1}+t_{F 2}+t_{R 1}+t_{R 2}\right)=I_{i} \cdot t_{U}
$$

for $i=1,2$. $\quad \lambda_{i}\left(t_{F 1}, t_{F 2}\right)$ is the expectation of the number of vehicles arriving during one period on side $i$ of the bottleneck when being controlled with times of open passage $\left(t_{F 1}, t_{F 2}\right), i=1,2$.

For $i=1,2$ let $q_{j}^{(i)}$ denote the distribution of the random variable $L^{(i)}\left(j \cdot t_{U}\right):(\Omega, \mathcal{A}, P) \rightarrow \mathbb{Z}_{+}(j=0,1, \ldots)$. Let $\mathcal{M}^{1}\left(\mathbb{Z}_{+}\right)$be the set of all probability measures on $\mathbb{Z}_{+}$, while $\pi_{\lambda^{\prime}}$ denotes the Poisson distribution with parameter $\lambda^{\prime}>0$.

The sequence $\left(q_{j}^{(i)}\right)_{j=0}$ satisfies the recursion

$$
q_{j+1}^{(i)}=T_{i} q_{j}^{(i)} \quad\left(j \in \mathbb{Z}_{+}\right)
$$

with the operator $T_{i}: \mathcal{M}^{1}\left(\mathbb{Z}_{+}\right) \rightarrow \mathcal{M}^{1}\left(\mathbb{Z}_{+}\right)$being defined by

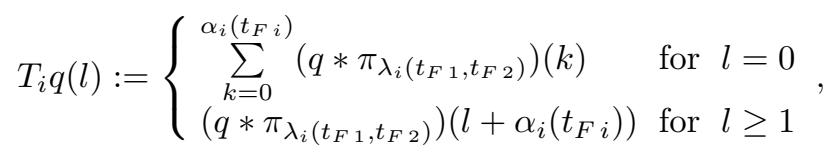

$i=1,2$, for details cp. [2].

From this recursion for the distributions $q_{j}^{(i)}$ of $L^{(i)}\left(j t_{U}\right)$ it follows that

$$
\int L^{(i)}\left(j t_{U}\right) d P, \quad(i=1,2)
$$

can be computed iteratively for any fixed $j \in \mathbb{Z}_{+}$.

We now give the definition of the waiting times which are of interest in connection with establishing an objective function. 
Definition 1 The waiting time until time $t$ for side $i \in\{1,2\}$ is defined by

$$
V^{(i)}(t):=\int_{0}^{t} L^{(i)}(s) d s
$$

For $j \in \mathbb{Z}_{+}$we denote by

$$
v_{j}^{(i)}:=\int_{j t_{U}}^{(j+1) t_{U}} L^{(i)}(s) d s
$$

the waiting time in the $(j+1)$-th control period for side $i$.

The total waiting time until time $t$ at the bottleneck is given by

$$
V^{\text {tot }}(t):=V^{(1)}(t)+V^{(2)}(t)=\int_{0}^{t} L^{(1)}(s)+L^{(2)}(s) d s .
$$

\section{Preparatory Results and McNeil's Formula}

In this and the next section we focus on an arbitrary but fixed side $i \in\{1,2\}$ at the bottleneck and omit the index $i$ in the definition of the waiting times and the arrival and queueing processes. For a fixed pair $\left(t_{F 1}, t_{F 2}\right)$ we define

$$
\lambda:=\lambda_{i}\left(t_{F 1}, t_{F 2}\right)
$$

and

$$
\alpha:=\alpha_{i}\left(t_{F i}\right) .
$$

Moreover, we write for brevity

$$
L_{j}:=L\left(j t_{U}\right)
$$

For the Markov chain $\left(L_{j}\right)$ that describes the process of queue lengths for side $i$ at the end of the time of free passage, it is shown in [2], 4.1 that

$$
\alpha \leq \lambda \Longrightarrow \lim _{j \rightarrow \infty} E\left(L_{j}\right)=\infty
$$

and

$$
\alpha>\lambda \Longrightarrow \sup _{j \in \mathbb{Z}_{+}} E\left(L_{j}\right)<\infty
$$

which may serve as a criterion for the occurrence of a traffic collapse.

Furthermore, if $\alpha>\lambda$, then the sequence of distributions $\left(P_{L_{j}}\right)$ converges with respect to the variational distance to a unique equilibrium distribution 
with finite expectation (cf. [3], 4.6). If we describe this equilibrium by a random variable $L: \Omega \rightarrow \mathbb{Z}_{+}$the results in [3] imply that

$$
\lim _{j \rightarrow \infty} E\left(L_{j}\right)=E(L)<\infty .
$$

Notice, (23) states more than only the well-known weak convergence of the Lindley process, but also the convergence of the sequence of expectations and the finiteness of the expectation of the equilibrium distribution.

In order to define an objective function in terms of the waiting times at the bottleneck we are interested in the asymptotic behaviour of the waiting times. McNeil proved a very helpful formula which connects the expected waiting time in a control period with the expected queue length.

\subsection{McNeil's Formula}

The expected waiting time in the $(j+1)$-th control period is a continuous function $F: \mathbb{R}_{+} \rightarrow \mathbb{R}_{+}$of the expected queue length at the end of the $j-$ th control period,

$$
E\left(v_{j}\right)=F\left(E\left(L_{j}\right)\right) .
$$

$F$ has the property

$$
\lim _{x \rightarrow \infty} F(x)=\infty,
$$

for details about the function $F$ see [4].

From this and (20) it easily follows

$$
\lim _{j \rightarrow \infty} E\left(v_{j}\right)=F(E(L))<\infty
$$

in the case $\alpha>\lambda$. Obviously, we obtain

$$
\lim _{j \rightarrow \infty} E\left(v_{j}\right)=\infty
$$

in the case $\alpha \leq \lambda$ having (21) and 3.1 in mind. Consequently, the occurrence or non-occurrence of a traffic collapse may be equivalently described in terms of the limit of expectations of waiting times.

\section{Laws of Large Numbers}

For the purpose of preparation of a theorem of the Little type we now focus on proving laws of large numbers for the sequences of queue lengths and 
waiting times. Notice, the theorem of the Little type in section 5 as well as the strong law of large numbers presented in this section do not coincide with standard results of queueing and Markov theory.

As in the previous section we omit the index $i$ while discussing the queueing process on the fixed side $i \in\{1,2\}$ and use the notations (18)-(20). Moreover, for this section we assume that $t_{F}$ is chosen in a way that $\alpha>\lambda$ is satisfied.

For the waiting time in the control period $j$ we have by definition of $v_{j}$ and the queueing process $(L(t))_{t \geq 0}$

$$
v_{j}=\int_{j t_{U}}^{(j+1) t_{U}} L(s) d s=\int_{j t_{U}}^{(j+1) t_{U}}\left(L_{j}+N\left(\left(j t_{U}, s\right]\right)-\alpha(s)\right)_{+} d s .
$$

Formally we can write

$$
v_{j}=g\left(L_{j},\left(N\left(\left(j t_{U}, t\right]\right)\right)_{j t_{U}<t \leq(j+1) t_{U}}\right)
$$

with a measurable function $g$; this means $v_{j}$ is a function of $L_{j}$ and the process of arrivals in the control period $j$, which is given by a Poisson process. Because of the independence of the arrival processes $\left(N\left(\left(j t_{U}, t\right]\right)\right)_{j t_{U}<t \leq(j+1) t_{U}}$ for $j \in \mathbb{Z}_{+}$it follows that $\left(L_{j}, v_{j}\right)$ is a Markov chain where the distribution of $v_{j}$ depends on random variables $\left(L_{k}, v_{k}\right)_{k=1}^{j-1}, L_{j}$ only by the random variable $L_{j}$.

We are now interested in ergodic theorems for $\left(L_{j}, v_{j}\right)$ that imply the validity of laws of large numbers.

For this purpose we use the setup and results given by M. Duflo in [1], chapter 8. Following theorem 8.2.16 in [1] we have to find a so-called small set $C \in P\left(\mathbb{Z}_{+}\right) \otimes \mathcal{B}$ for which the return time

$$
T:=\inf \left\{j \in \mathbb{N} \mid\left(L_{j}, v_{j}\right) \in C\right\}
$$

satisfies the condition

$$
\sup _{(n, x) \in C} E\left(T \mid\left(L_{0}, v_{0}\right)=(n, x)\right)<\infty
$$

For proving $C$ to be a small set for the chain $\left(L_{j}, v_{j}\right)$ it suffices to show the existence of a $\delta \in(0,1)$ and a probability measure $\xi$ concentrated on $C$ such that

$$
P\left(\left(L_{j+1}, v_{j+1}\right) \in \cdot \mid\left(L_{j}, v_{j}\right)=(n, x)\right) \geq \delta \cdot \xi(.)
$$

for all $(n, x) \in C$. We now want to prove this property for the set

$$
C:=\{0, \ldots, \alpha\} \times \mathbb{R} \in \mathcal{P}\left(\mathbb{Z}_{+}\right) \otimes \mathcal{B} .
$$


Theorem $1 C$ defined by (28) is a small set for the chain $\left(L_{j}, v_{j}\right)$.

Proof: Suppose $(n, x) \in C$, that is $n \leq \alpha$. If $\left(L_{j}, v_{j}\right)=(n, x)$ and no arrivals occur in the $(j+1)$-th control period, the recursion formula for the queue length implies $L_{j+1}=0$ because all $n \leq \alpha$ vehicles pass the bottleneck during the $(j+1)$-th control period. If furthermore no arrivals occur in the $(j+2)$-th control period then no vehicles are waiting during the whole control period, so it follows $v_{j+1}=0$.

Formally we obtain

$$
\begin{aligned}
& P\left(L_{j+1}=0 \mid\left(L_{j}, v_{j}\right)=(n, x)\right) \\
& =P\left(\left(n-\alpha+N\left(\left(j t_{U},(j+1) t_{U}\right]\right)\right)_{+}=0 \mid\left(L_{j}, v_{j}\right)=(n, x)\right) \\
& \geq P\left(N\left(\left(j t_{U},(j+1) t_{U}\right]\right)=0 \mid\left(L_{j}, v_{j}\right)=(n, x)\right) \\
& =e^{-I \cdot t_{U}}
\end{aligned}
$$

and

$$
\begin{aligned}
& P\left(v_{j+1}=0 \mid L_{j+1}=0,\left(L_{j}, v_{j}\right)=(n, x)\right) \\
& =P\left(v_{j+1}=0 \mid L_{j+1}=0\right) \\
& \left.\geq P\left(N\left((j+1) t_{U},(j+2) t_{U}\right]\right)=0 \mid L_{j+1}=0\right) \\
& =e^{-I \cdot t_{U}}
\end{aligned}
$$

Combining these inequalities we get

$$
P\left(\left(L_{j+1}, v_{j+1}\right)=(0,0) \mid\left(L_{j}, v_{j}\right)=(n, x)\right) \geq e^{-2 I \cdot t_{U}} .
$$

Setting $\delta:=e^{-2 I \cdot t_{U}}$ and $\xi$ the probability measure concentrated in $(0,0) \in$ $\mathbb{Z}_{+} \times \mathbb{R}$ completes the proof of (27) for the set $C$, which is therefore a small set.

We will use the so-called Pake's criterion in order to prove (26). For a proof the reader is referred to [1].

Theorem 2 (PAKe's CRITERION) Let $\Lambda: \mathbb{Z}_{+} \times \mathbb{R}_{+} \rightarrow \mathbb{R}_{+}$be a function and $h, R, K>0$ such that for all $(n, x) \in \mathbb{Z}_{+} \times \mathbb{R}_{+}$

$$
E\left(\Lambda\left(L_{j+1}, v_{j+1}\right) \mid\left(L_{j}, v_{j}\right)=(n, x)\right) \leq\left\{\begin{array}{lll}
\Lambda(n, x)-h & \text { if } \quad \Lambda(n, x)>R \\
K & \text { if } \quad \Lambda(n, x) \leq R
\end{array}\right.
$$

is valid. Then the return time

$$
T:=\inf \left\{j \in \mathbb{N} \mid\left(L_{j}, v_{j}\right) \in C\right\}
$$


satisfies

$$
E\left(T \mid\left(L_{0}, v_{0}\right)=(n, x)\right) \leq \frac{\Lambda(n, x)}{h}+\left(1+\frac{K}{h}\right)
$$

TheOREM 3 The return time $T:=\inf \left\{j \in \mathbb{N} \mid\left(L_{j}, v_{j}\right) \in C\right\}$ for the set $C$ defined in (28) satisfies

$$
\sup _{(n, x) \in C} E\left(T \mid\left(L_{0}, v_{0}\right)=(n, x)\right)<\infty
$$

Proof: Define the function

$$
\Lambda: \mathbb{Z}_{+} \times \mathbb{R} \rightarrow \mathbb{R}_{+} \quad \text { by } \Lambda(n, x):=n .
$$

Then $C=\{\Lambda \leq \alpha\}$. Having (9) in mind we obtain for $(n, x) \notin C$

$$
\begin{aligned}
& E\left(\Lambda\left(L_{j+1}, v_{j+1}\right) \mid\left(L_{j}, v_{j}\right)=(n, x)\right) \\
& \quad=E\left(L_{j+1} \mid\left(L_{j}, v_{j}\right)=(n, x)\right) \\
& \quad=E\left(n-\alpha+N\left(\left(j t_{U},(j+1) t_{U}\right]\right) \mid\left(L_{j}, v_{j}\right)=(n, x)\right) \\
& \quad=n-\alpha+\lambda \leq n-h=\Lambda(n, x)-h
\end{aligned}
$$

where $h:=\alpha-\lambda>0$ by the assumption $\alpha>\lambda$.

For $(n, x) \in C$ it follows from (9)

$$
\begin{aligned}
E\left(\Lambda\left(L_{j+1}, v_{j+1}\right) \mid\left(L_{j}, v_{j}\right)=(n, x)\right) & =E\left(L_{j+1} \mid\left(L_{j}, v_{j}\right)=(n, x)\right) \\
& \leq n+\lambda \\
& \leq \alpha+\lambda .
\end{aligned}
$$

Now we are able to apply Pake's criterion in theorem 2 with $h=\alpha-\lambda>0$, $R:=\alpha$ and $K:=\alpha+\lambda$ from which the proposition follows.

The proof of theorem 3 via Pake's criterion shows a little bit more than what is stated, because the validity of the condition in the criterion implies that the expectation of the return times is finite for all $(n, x) \in \mathbb{Z}_{+} \times \mathbb{R}$.

In particular, the set $C$ is reached from every point $(n, x)$ with probability 1. Having this and theorem 1 and 3 in mind we can apply theorem 8.2.16 and 8.3.18 from [1]. 
THEOREM 4 If $\alpha>\lambda$ the Markov chain $\left(L_{j}, v_{j}\right)$ is positive recurrent and converges independently of the initial distribution $P_{\left(L_{0}, v_{0}\right)}$ weakly to a random variable $(L, v)$. Moreover the following law of large numbers is valid:

$$
\frac{1}{m} \sum_{j=1}^{m} f\left(L_{j}, v_{j}\right) \rightarrow E(f(L, v)) \quad P-\text { a.s. }
$$

for a measurable function $f: \mathbb{Z}_{+} \times \mathbb{R} \rightarrow \mathbb{R}$ with $E(f(L, v))$ being finite.

As stated in (23), we already know from [3] that $E(L)<\infty$. The result of McNeil, given by 3.1 , easily implies

$$
E(v)=F(E(L))<\infty
$$

in this case. We therefore obtain with the help of theorem 4.7

$$
\begin{array}{ll}
\frac{1}{m} \sum_{j=1}^{m} L_{j} \rightarrow E(L) & P-\text { a.s. } \\
\frac{1}{m} \sum_{j=1}^{m} v_{j} \rightarrow E(v) & P-\text { a.s. }
\end{array}
$$

This completes the proof of laws of large numbers for the queue length and the waiting time in one control period. For the waiting time until time $t$ we can now prove the following result.

Corollary 1 If $\alpha>\lambda$ then

$$
t^{-1} V(t) \rightarrow \frac{E(v)}{t_{U}} \quad P-a . s .
$$

Proof: By definition of $V(t)$ and $v_{j}$ (see (15) and (16)) we have for $m \in \mathbb{Z}_{+}$

$$
V\left(m t_{U}\right)=\sum_{j=1}^{m} v_{j}
$$


For $t \in \mathbb{R}_{+}$let $m(t)$ be the largest integer such that $m(t) \cdot t_{U}$ is less or equal $t$. Then we obtain

$$
\begin{aligned}
t^{-1} V(t)= & \frac{m(t) \cdot t_{U}}{t} \cdot \frac{1}{m(t) \cdot t_{U}}\left(V\left(m(t) \cdot t_{U}\right)+V(t)\right. \\
& \left.-V\left(m(t) \cdot t_{U}\right)\right) \\
= & \frac{m(t) \cdot t_{U}}{t} \cdot\left(\frac{1}{m(t) \cdot t_{U}} V\left(m(t) \cdot t_{U}\right)\right. \\
& \left.+\frac{1}{m(t) \cdot t_{U}}\left(V(t)-V\left(m(t) \cdot t_{U}\right)\right)\right)
\end{aligned}
$$

By definition of $m(t)$,

$$
\frac{m(t) \cdot t_{U}}{t} \rightarrow 1, \quad t \rightarrow \infty
$$

From (i) and (30) we get

$$
\frac{1}{m(t) \cdot t_{U}} V\left(m(t) t_{U}\right)=t_{U}^{-1} \cdot \frac{1}{m(t)} \sum_{j=1}^{m(t)} v_{j} \rightarrow t_{U}^{-1} E(v) \quad P-\text { a.s. (iv) }
$$

For $m(t) \cdot t_{U} \leq t \leq(m(t)+1) t_{U}$ it follows

$$
V(t)-V\left(m(t) \cdot t_{U}\right)=V(t)-\sum_{j=1}^{m(t)} v_{j} \leq v_{m(t)+1}
$$

so having the a.s. convergence of $m^{-1} \sum v_{j}$ in mind we are able to deduce

$$
\frac{1}{m(t) \cdot t_{U}}\left(V(t)-V\left(m(t) \cdot t_{U}\right)\right) \leq \frac{1}{m(t) \cdot t_{U}} v_{m(t)+1} \rightarrow 0, \quad t \rightarrow \infty .
$$

Now (ii) - (v) imply

$$
t^{-1} V(t) \rightarrow t_{U}^{-1} E(v), \quad t \rightarrow \infty .
$$

\section{A Theorem of the Little Type and Minimal Individual Wait- ing Times}

With the help of the law of large numbers in corollary 1 we are in the situation to prove a theorem of the Little type for both sides of the bottleneck, which does not follow from standard queueing and Markov theory. 
In the sequel we do no longer focus on only one side so that we use the index $i$ again as in section 2 . In the case $\alpha_{i}\left(t_{F i}\right)>\lambda_{i}\left(t_{F 1}, t_{F 2}\right)$ we denote by

$$
L^{(i)} \text { and } v^{(i)}
$$

the (according to theorem 4 existing) limiting random variables for the queue length and waiting time in a control period for side $i$, respectively.

We now denote by $W_{n}^{(i)}$ the waiting time of the $n$-th vehicle arriving at side $i$. Because the queueing system at side $i$ is FIFO and we work with a Poisson arrival process with intensity $I_{i}$, the following lemma is an easy corollary from the results of S. Stidham and M. El Taha [5] and corollary 1 .

LEMMA 1 If $\alpha_{i}\left(t_{F i}\right)>\lambda_{i}\left(t_{F 1}, t_{F 2}\right)$, it follows

$$
\lim _{m \rightarrow \infty} \frac{1}{m} \sum_{j=1}^{m} W_{n}^{(i)}=\frac{E\left(v^{(i)}\right)}{I_{i} \cdot t_{U}} .
$$

P-a.s.

This result is a theorem of the Little type in the situation that the queueing process on each side is analyzed separately. For the purpose of defining an objective function, taking the average over all waiting times of vehicles arriving at the bottleneck is of even higher interest. We therefore define by $W_{n}$ the waiting time of the $n-t h$ vehicle arriving at the bottleneck, regardless on which side. In preparation of a theorem of the Little type for this waiting times we interpret the queueing process at the bottleneck as an input-output system with arrival process $A^{\text {tot }}$ which is the superposition of $A^{(1)}$ and $A^{(2)}$ defined by

$$
A_{t}^{\text {tot }}:=A_{t}^{(1)}+A_{t}^{(2)} \quad\left(t \in \mathbb{R}_{+}\right) .
$$

This is again a Poisson process with intensity $I_{1}+I_{2}$. The process of the queue length in this system is given by

$$
L^{\text {tot }}(t):=L^{(1)}(t)+L^{(2)}(t) \quad\left(t \in \mathbb{R}_{+}\right) .
$$

Of course, we have an equivalent to corollary 1 for this queuing system because we have for the total waiting time defined by (15)

$$
\begin{aligned}
\frac{1}{t} V^{\text {tot }}(t) & =\frac{1}{t}\left(V^{(1)}(t)+V^{(2)}(t)\right) \\
& =\frac{1}{t} \int L^{\operatorname{tot}}(s) d s \rightarrow \frac{E\left(v^{(1)}\right)+E\left(v^{(2)}\right)}{t_{U}}
\end{aligned}
$$

$P$-a.s. as $t$ goes to infinity. Obviously, the queueing discipline is now no longer FIFO. The $(n+1)$-th arriving vehicle may arrive on side 1 and leave 
the bottleneck earlier than the $n$-th vehicle arriving on side 2 . But with the help of lemma 5.2 and corollary 5.50 from [5] it is possible to prove a theorem of the Little type in this setup, too.

TheOREM 5 If $\alpha_{i}\left(t_{F i}\right)>\lambda_{i}\left(t_{F 1}, t_{F 2}\right)$ then

$$
\lim _{m \rightarrow \infty} \frac{1}{m} \sum_{n=1}^{m} W_{n}=\frac{E\left(v^{(1)}\right)+E\left(v^{(2)}\right)}{\left(I_{1}+I_{2}\right) \cdot t_{U}} .
$$

P-a.s.

Proof: Following [5], corollary 5.50, it suffices to show that

$$
\lim _{n \rightarrow \infty} \frac{1}{n} W_{n}=0
$$

$P$-a.s., in this case the assertion follows from (40) and the fact that the arrival process $A^{\text {tot }}$ is Poisson with intensity $I_{1}+I_{2}$.

Let us assume that $\omega \in \Omega$ such that $n^{-1} W_{n}(\omega)$ does not converge to 0 as $n$ goes to infinity. Then there exists an $\epsilon>0$ and a sequence $\left(n_{k}\right)$ such that

$$
W_{n_{k}}(\omega) \geq \epsilon \cdot n_{k} \quad(k \in \mathbb{N}) .
$$

The $n_{k}$-th arrival at the bottleneck now corresponds to an arrival on one side, which we denote by $i_{k}$, and we assume that this is the $m_{k}-$ th arrival on this side. From (ii) we get

$$
W_{m_{k}}^{\left(i_{k}\right)}(\omega) \geq \epsilon \cdot n_{k} \geq \epsilon \cdot m_{k} \quad(k \in \mathbb{N}) .
$$

But (iii) implies that at one side $i \in\{1,2\}$ there exists a sequence $\left(m_{k}^{\prime}\right)$ such that

$$
W_{m_{k}^{\prime}}^{(i)}(\omega) \geq \epsilon \cdot m_{k}^{\prime} .
$$

From this we obtain by straightforward analysis that the limit

$$
\lim _{m \rightarrow \infty} \frac{1}{m} \sum_{n=1}^{m} W_{n}^{(i)}(\omega)
$$

cannot exist. But we already know from lemma 1 that the limes of (v) exists for almost every $\omega \in \Omega$, so (i) must be satisfied for almost every $\omega \in \Omega$ which completes the proof.

With theorem 5 we are in the situation to define in corollary 2 the mean individual waiting time, 
Corollary 2 Let be $\alpha_{i}\left(t_{F i}\right)<\lambda_{i}\left(t_{F 1}, t_{F 2}\right)(i=1,2)$. The almost sure limit

$$
\bar{W}:=\frac{E\left(v^{(1)}\right)+E\left(v^{(2)}\right)}{\left(I_{1}+I_{2}\right) \cdot t_{U}}
$$

of the sequence $\left(m^{-1} \sum W_{n}\right)$ is called mean individual waiting time at the bottleneck.

By (29),

$$
\bar{W}=\frac{F\left(E\left(L^{(1)}\right)\right)+F\left(E\left(L^{(2)}\right)\right)}{\left(I_{1}+I_{2}\right) \cdot t_{U}} .
$$

We now define a pair $\left(t_{F 1}^{*}, t_{F 2}^{*}\right)$ to be of minimal waiting time, if it minimizes the mean individual waiting time at a bottleneck.

\section{Definition 2}

2.1 A pair $\left(t_{F 1}, t_{F 2}\right)$ is called ergodic, iff

$$
\alpha_{i}\left(t_{F i}\right)<\lambda_{i}\left(t_{F 1}, t_{F 2}\right)(i=1,2) .
$$

2.2 An ergodic pair $\left(t_{F 1}^{*}, t_{F 2}^{*}\right)$ is of minimal waiting time, iff

$$
\bar{W}=\frac{E\left(v^{(1)}\right)+E\left(v^{(2)}\right)}{\left(I_{1}+I_{2}\right) \cdot t_{U}}
$$

as a function of $\left(t_{F 1}, t_{F 2}\right)$ has a minimum in $\left(t_{F 1}^{*}, t_{F 2}^{*}\right)$.

Remark 1 Because the intensities $I_{1}$ and $I_{2}$ are assumed to be fixed, a minimization of the mean individual waiting time is equivalent to the minimization of the almost sure limit

$$
\frac{E\left(v^{(1)}\right)+E\left(v^{(2)}\right)}{t_{U}}
$$

of $\left(\frac{1}{t} V^{\text {tot }}(t)\right)_{t \in \mathbb{R}_{+}}($see $(40))$; which may be interpreted as a minimization of the mean total waiting time. Let now $D_{t}^{\text {tot }}$ denote the total number of vehicles that have left the bottleneck until time $t$ defined by

$$
D_{t}^{\mathrm{tot}}:=A_{t}^{\mathrm{tot}}-L_{t}^{\mathrm{tot}}
$$

We then call $\left(D_{t}^{\text {tot }}\right)_{t \in \mathbb{R}_{+}}$the departure process for the queueing system at the bottleneck. 
As the realizations of the arrival process $A^{\text {tot }}$ cannot be influenced by the administrator of the bottleneck, the expression

$$
\liminf _{t \rightarrow \infty} \frac{1}{t}\left(\int_{0}^{t} D_{s}^{\mathrm{tot}} d s-\int_{0}^{t} A_{s}^{\mathrm{tot}} d s\right)
$$

may be interpreted as a measure of the throughput per time unit of the special setting of times of open passage. This gives rise for the following definition.

Definition 3 An ergodic pair $\left(t_{F 1}^{*}, t_{F 2}^{*}\right)$ is of maximal throughput per time unit iff

$$
\liminf _{t \rightarrow \infty} \frac{1}{t}\left(\int_{0}^{t} D_{s}^{\mathrm{tot}} d s-\int_{0}^{t} A_{s}^{\mathrm{tot}} d s\right)
$$

as a function of $\left(t_{F 1}, t_{F 2}\right)$ has $P-$ a.s. a maximum in $\left(t_{F 1}^{*}, t_{F 2}^{*}\right)$.

The optimization problems corresponding to the definitions 2.2 and 3 are equivalent in the following sense.

THEOREM 6 An ergodic pair $\left(t_{F 1}^{*}, t_{F 2}^{*}\right)$ is of maximal throughput per time unit iff it is of minimal waiting time.

Proof: From the definition (48) we get a.s.

$$
\begin{aligned}
\liminf _{t \rightarrow \infty} \frac{1}{t}\left(\int_{0}^{t} D_{s}^{\mathrm{tot}} d s-\int_{0}^{t} A_{s}^{\mathrm{tot}} d s\right) & =\liminf _{t \rightarrow \infty} \frac{1}{t} \int_{0}^{t}-L_{s}^{\mathrm{tot}} d s \\
& =-\lim _{t \rightarrow \infty} \frac{1}{t} \int_{0}^{t} L_{s}^{\mathrm{tot}} d s \\
& =-\lim _{t \rightarrow \infty} \frac{1}{t} V^{\mathrm{tot}}(t) .
\end{aligned}
$$

The assertion follows therefore from remark 1.

Example 1 Corollary 2 allows to approximate the mean individual waiting time of a bottleneck system within a stochastic computer experiment by taking the average of the waiting times of all vehicles. On the other hand it is possible to determine the mean individual waiting time for one side numerically using recursion (12) in order to approximate the equilibrium distribution of the queue length and then apply (47). The following figures show the contours of the mean individual waiting time as a function of $t_{F 1}$ and $t_{F 2}$, connecting points with the same numerical waiting time values derived by stochastic experimentation on the computer (FIG.2) and numerically with the help of the recursion (12) of the distributions (FIG.3). 
These values were systematically determined for various points $\left(t_{F 1}, t_{F 2}\right)$ of times of free passage. The pair $\left(t_{F 1}^{*}, t_{F 2}^{*}\right)$ of minimal waiting time is marked in both cases.

The technical parameters for the generation of FIG.2 and FIG.3 had been chosen to be

$$
\begin{aligned}
& \Delta_{1}=\Delta_{2}=\frac{1250}{3600} \mathrm{veh} / \mathrm{s} \\
& I_{1}=0.125 \mathrm{veh} / \mathrm{s}, \quad I_{2}=0.1 \mathrm{veh} / \mathrm{s} \\
& t_{R 1}=t_{R 2}=30 \mathrm{~s}
\end{aligned}
$$

Evidently, the results do not differ a lot, so the stochastic experimentation proves to be a good approximation of the numerical results as it was predicted by the proved law of large numbers.

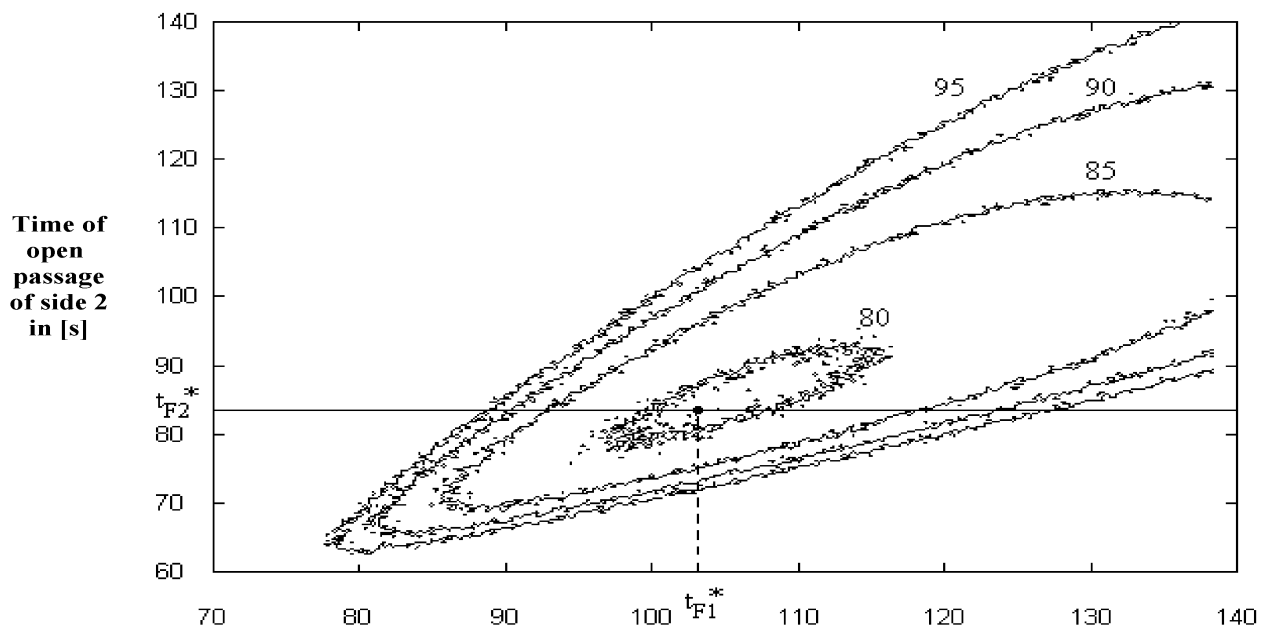

Figure 2. Time of open passage side 1 in $[\mathrm{s}]$

Figure 4 shows the value of the waiting time for varying time of open passage $t_{F 1}$ for side 1 and fixed time of open passage $t_{F 2}=t_{F 2}^{*}$ for side 2 of the bottleneck for both stochastic experimentation and numerical determination. In Figure 2 and Figure 3 the pairs of times of open passage corresponding to this figure are marked by a solid line. Again the results show, that the values determined by the computer experiment are close to the values determined numerically using the recursion of distributions. 


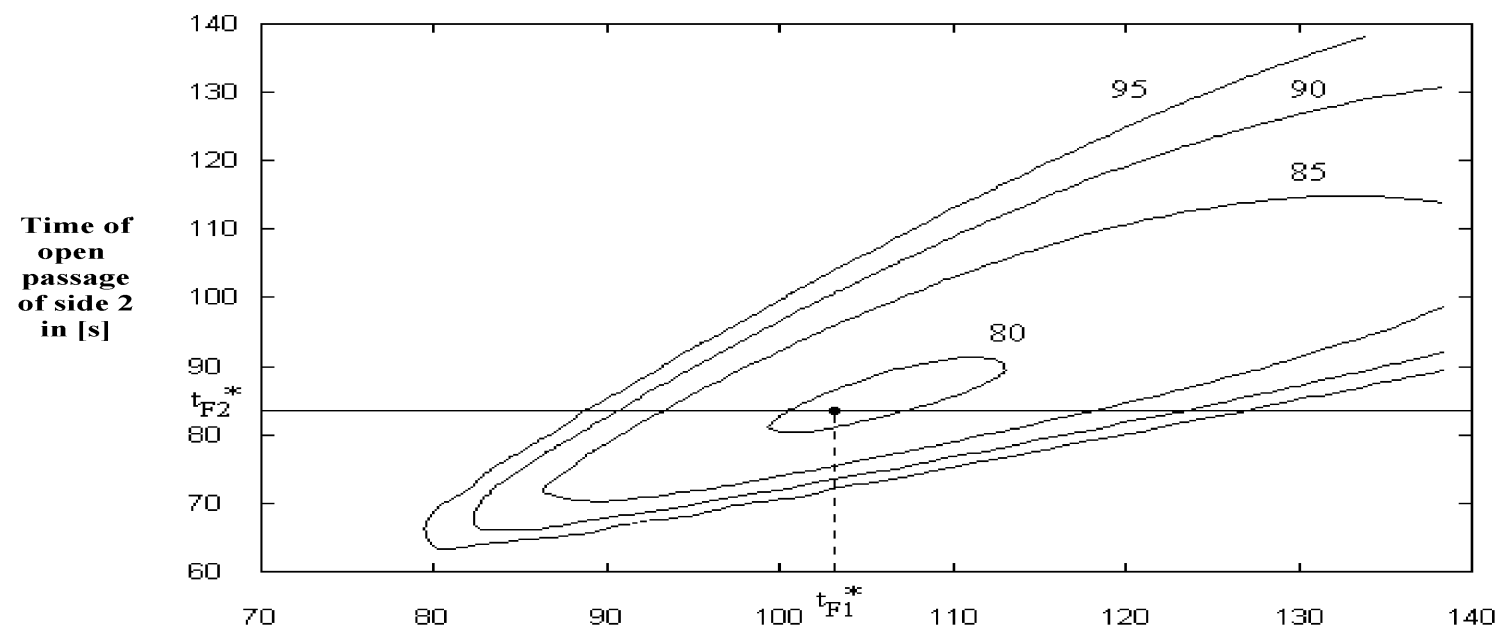

Figure 3. Time of open passage side 1 in [s]

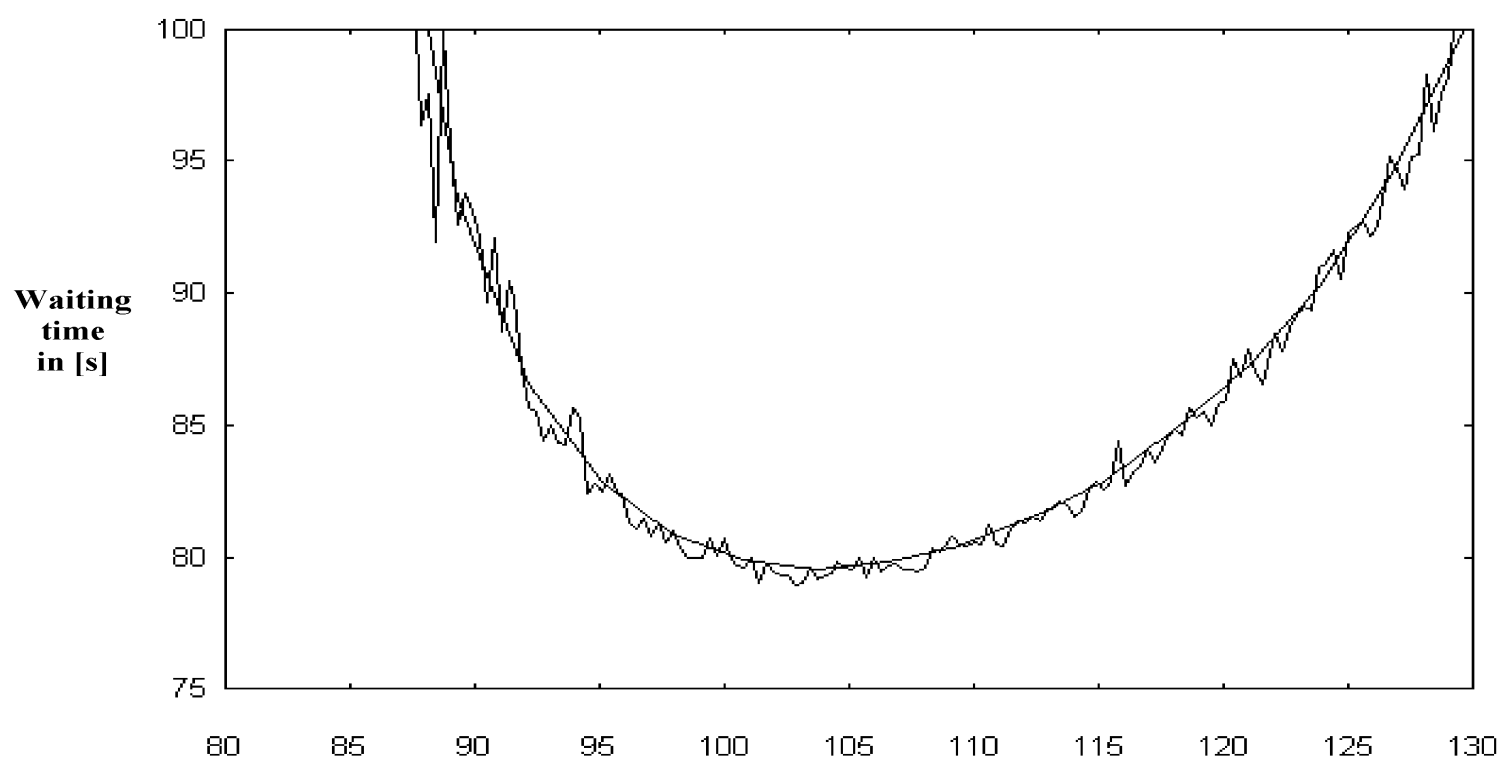

Figure 4. Time of open passage side 1 in [s] 


\section{References}

1. M. Duflo. Random Iterative Models Applications of Mathematics 34, SpringerVerlag, Berlin, Heidelberg, New York, 1997.

2. E. Grycko, O. Moeschlin. A Criterion for the Occurrence or Non-occurrence of a Traffic Collapse at a Bottleneck. Commun. Statist. - Stochastic Models, 14(3), 1998 pp. 571-584.

3. E. Grycko, O. Moeschlin. A Concept of Optimal Control of a Bottleneck with Symmetric Volume of Traffic. Commun. Statist. - Stochastic Models, 14(3), 1998 pp. 585-600.

4. D. McNeil. A Solution to the Fixed-Cycle Traffic Light Problem for Compound Poisson Arrivals Journal of Applied Probability, 5, 1968 pp. 624-635.

5. S. Stidham, M. El-Taha. Sample-path techniques in queueing theory Advances in queueing, pp. 119-166, Probab. Stochastics Ser., CRC, Boca Raton, 1995. 


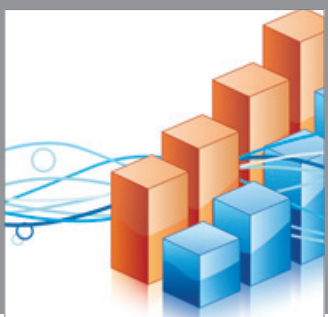

Advances in

Operations Research

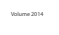

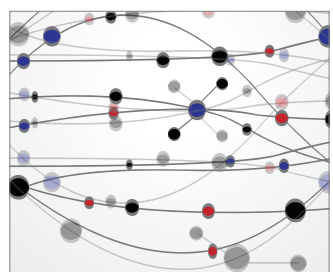

\section{The Scientific} World Journal
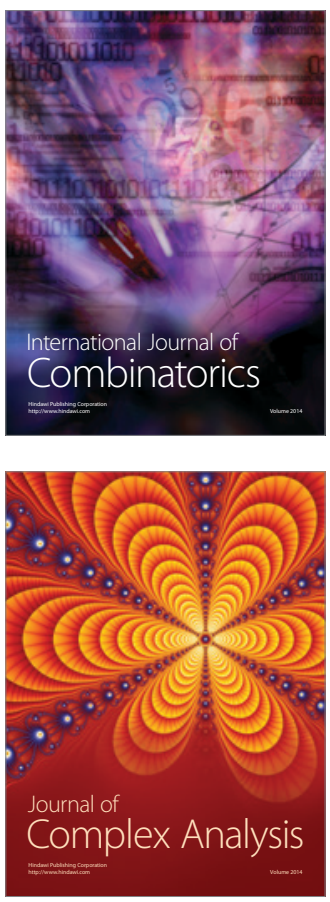

International Journal of

Mathematics and

Mathematical

Sciences
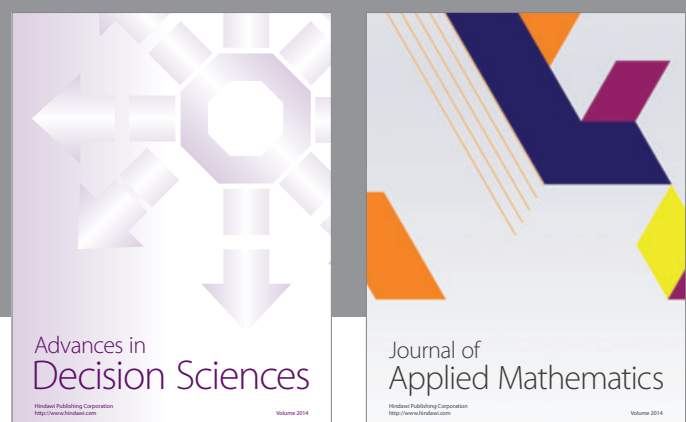

Journal of

Applied Mathematics
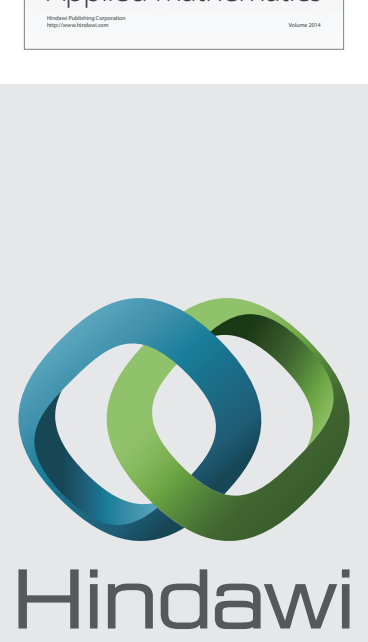

Submit your manuscripts at http://www.hindawi.com
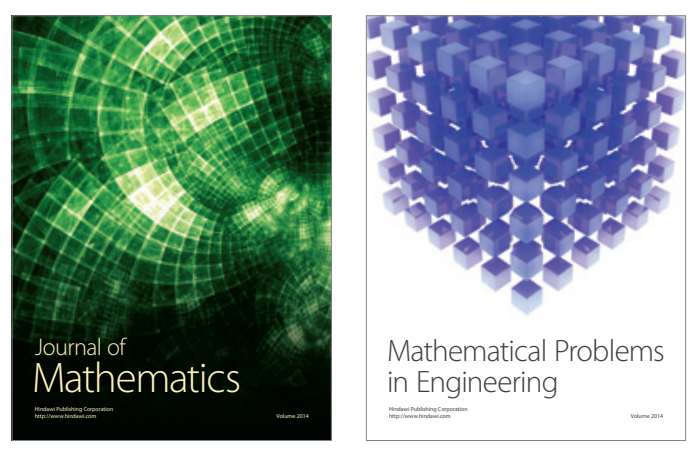

Mathematical Problems in Engineering
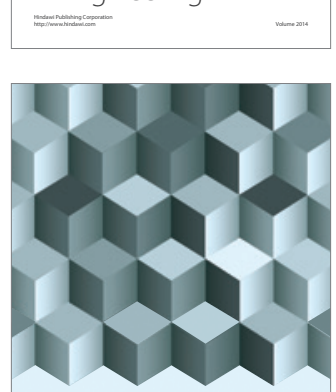

Journal of

Function Spaces
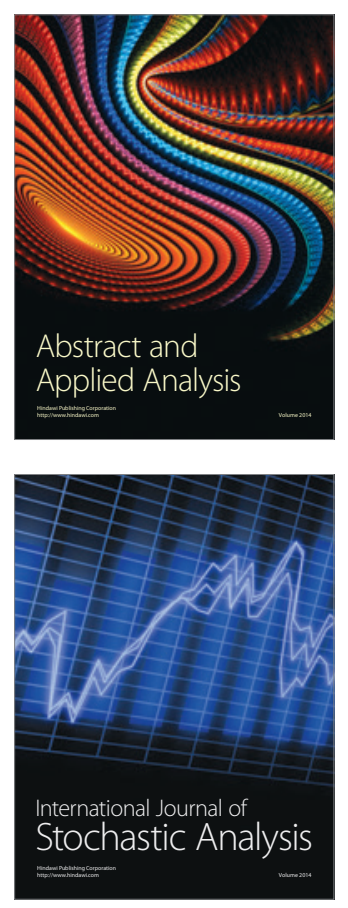

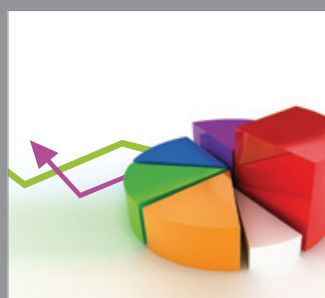

ournal of

Probability and Statistics

Promensencen
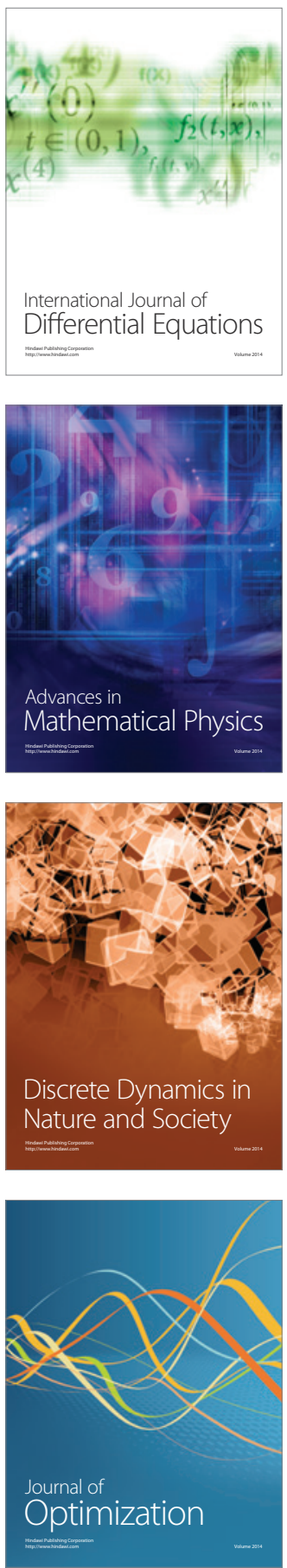